\title{
Impact of Surface Treatment Methods on Bond Strength of Orthodontic Brackets to Indirect Composite Provisional Restorations
}

\author{
Sary Borzangy
}

\begin{abstract}
Aim: To assess the impact of different surface treatment protocols on the shear bond strength (SBS) of metal orthodontic brackets (MOBs) and ceramic orthodontic brackets (CBOs) bonded to provisional crowns via the SBS test.

Materials and methods: A total of 120 provisional indirect composite crowns (SR Nexco; Ivoclar Vivadent) for maxillary first premolars were fabricated and evenly allocated into two groups: $\mathrm{MOBs}$ and $\mathrm{CBO}$. According to the surface treatment protocol, each group was divided into three subgroups: group CO, no treatment; group HF, the surface was etched with $10 \%$ hydrofluoric acid; and group SA, the surface was sandblasted followed by silanization. After bracket bonding, the samples were subjected to 3,000 thermocycles between $5^{\circ} \mathrm{C}$ and $55^{\circ} \mathrm{C}$. SBS was evaluated using a universal testing machine at a crosshead speed of $1 \mathrm{~mm} /$ minute and the adhesive remnant index (ARI) was identified. For statistical analysis, ANOVA and Tukey HSD post hoc tests were performed.

Results: Mean bond strength values for $\mathrm{CBO}$ cemented to control, HF-, and SA-treated subgroups before and after thermocycling were $(9.6 \pm 1.4,6.2 \pm 1.1),(17.8 \pm 2.1,13.8 \pm 1.3)$, and $(17.2 \pm 1.4,12.1 \pm 1.8) \mathrm{MPa}$, respectively. For the metallic brackets, the results were $(7.7 \pm 2.3$, $3.9 \pm 1.4),(15.5 \pm 1.6,12.8 \pm 1.2)$, and $(15 \pm 1.2,11.2 \pm 1.6) \mathrm{MPa}$, respectively. There was a significant difference $(p=0.000)$ between ceramic and metallic bracket groups.

Conclusion: Conditioning of indirect composite provisional crowns either with HF or SA was significantly affecting the adhesion to both bracket types.

Clinical significance: Increasing the bond strength between provisional crowns and orthodontic brackets (OBs) may improve the treatment standard provided to patients.

Keywords: Adhesion, Indirect composite, Orthodontic brackets, Provisional crowns, Surface conditioning.

The Journal of Contemporary Dental Practice (2019): 10.5005/jp-journals-10024-2696
\end{abstract}

\section{INTRODUCTION}

Orthodontics is oftentimes an intermediate stage to oral rehabilitation. ${ }^{1}$ Thus, abutment teeth often have provisional crowns during orthodontic treatment until the teeth have been moved into more favorable positions to receive the definitive crowns. ${ }^{2}$ Treatment with provisional restorations must provide positional stability for abutment teeth, esthetics, reestablish masticatory and occlusal functions, adequate marginal adaptation, and maintain periodontal health. ${ }^{2-5}$

Although chemically activated resins are preferred by many clinicians for provisional crowns fabrication to provide adequate short-term interim prostheses, the use of visible light polymerized indirect processed composite restorations is often recommended as a long-term provisional restoration until the final treatment is indicated. ${ }^{1,6-9}$

Many researchers reported that the conventional bonding protocol could not provide sufficient adhesion to provisional restorations to withstand orthodontic forces, and orthodontists are often challenged with bonding attachments to these provisional restorations. ${ }^{7}$ Several approaches attempted to improve the surface roughness and the bonding surface area. ${ }^{1,2,7,10-12}$ The approaches suggested can be classified into two groups: mechanical or chemical. Mechanical methods involve sandblasting and surface grinding with a silica carbide paper or a diamond bur. ${ }^{13-15}$ The chemical method is achieved by hydrofluoric acid etching and a silane primer application. ${ }^{16,17}$
Department of Substitutive Dental Sciences, College of Dentistry, Taibah University, Medina, Kingdom of Saudi Arabia

Corresponding Author: Sary Borzangy, Department of Substitutive Dental Sciences, College of Dentistry, Taibah University, Medina, Kingdom of Saudi Arabia, Phone: +966 504358228, e-mail: sborzangy@ gmail.com

How to cite this article: Borzangy S. Impact of Surface Treatment Methods on Bond Strength of Orthodontic Brackets to Indirect Composite Provisional Restorations. J Contemp Dent Pract 2019;20(12):1412-1416.

Source of support: Nil

Conflict of interest: None

The bond between provisional materials and OBs is affected by different factors, including the type of adhesive material, the provisional material, the storage time after bonding, and materials aging. ${ }^{1,2,15,18,19}$ The strength of this bond should prevent the failure caused by the orthodontic forces, mastication, and other oral functions. During the treatment period, the techniques and materials used in bonding should maintain the brackets bonded and keep the crown's surface intact during debonding. The aim of this study was to investigate the impact of various surface treatment protocols and thermocycling on the SBS of MOBs and COBs to indirect composites provisional crowns in vitro. It was hypothesized

(c) The Author(s). 2019Open Access This article is distributed under the terms of the Creative Commons Attribution 4.0 International License (https://creativecommons. org/licenses/by-nc/4.0/), which permits unrestricted use, distribution, and non-commercial reproduction in any medium, provided you give appropriate credit to the original author(s) and the source, provide a link to the Creative Commons license, and indicate if changes were made. The Creative Commons Public Domain Dedication waiver (http://creativecommons.org/publicdomain/zero/1.0/) applies to the data made available in this article, unless otherwise stated. 
that SBS values would differ significantly among the different conditioning techniques.

\section{Materials and Methods}

\section{Samples Preparation}

This experimental study was approved by the Research Ethical Committee of the College of Dentistry, Taibah University, Madinah, Kingdom of Saudi Arabia (approval \# TUCDREC/20190406/ SSBorzangy). The study included 120 human permanent maxillary sound first premolars freshly extracted for clinical reasons. The obtained teeth were cleaned and stored for 6 months after extraction in $0.5 \%$ chloramine solution at $4^{\circ} \mathrm{C}$. Chemically polymerized acrylic resin (Sampl Kwick, Buehler, Lake Bluff, IL, USA) was used to encircle the root of these teeth. Standardized tooth preparations for all the teeth were done using a lathe cut machine to stimulate the preparation for all-ceramic crowns. Impressions were made using the vinylpolysiloxane addition-cured silicone impression material (Elite HD, Zhermack Spa, Via Bovazecchino, Italy) for each prepared tooth and poured with dental stone type IV consistent with manufacturer's recommendations.

An indirect composite material (SR Nexco, Ivoclar Vivadent AG, Bendererstrasse 2, FL-9494 Schaan) was used in this study for the fabrication of provisional crowns. The manufacturer's instructions were followed to mix the materials and inject them into a silicone index simulating the full form of the premolar tooth mold. It was held under compression and then polymerized by a visible light cure system for 90 seconds. The provisional crowns were finished by using rubber polishers and silicone polishing wheels followed by polishing with a universal polishing past and goat hairbrush following manufacturer's recommendations. Each provisional crown was cemented to its corresponding tooth using non-eugenol temporary cement (Temp Bond NE, Kerr Corp, Orange, CA, USA), for 5 minutes under $5 \mathrm{~kg}$ static load.

\section{Group Classification}

Grounding of the bonded area of composite surfaces was done with a 600-grit silicon carbide $(\mathrm{SiC})$ paper under running water for 30 seconds to expose filler particles except for the control group. ${ }^{10}$ The samples were allocated into two groups $(n=60)$ according to the type of OBs used: group MOBs and group COBs. These groups were further subdivided into three subgroups $(n=20)$ according to the surface treatment protocol used on the indirect composite provisional crown. Group CO: was without any surface treatment. Group HF: 10\% hydrofluoric acid (Dentsply Caulk, Milford, DE, USA) was used for etching for 30 seconds and then samples were washed under running water and ultrasonically cleaned in distilled water for 5 minutes. The silane primer (Porcelain Prep-Kit, Pulpdent, Watertown, MA, USA) was applied to the composite samples, left to evaporate for 3 minutes, and then air-dried for 30 seconds following manufacturer's instructions. Group SA: sandblasting with $50 \mu \mathrm{m}$ aluminum oxide particles (Danville Eng. Inc., San Ramon, CA, USA) was done at a pressure of 2.8 bar for about 10 seconds from a distance nearly $10 \mathrm{~mm}$; the residues of sand particles were removed gently with air and silane solution was then applied as described before.

\section{Bracket Bonding}

Sixty maxillary premolar COBs (Victory series 3M Unitek, Monrovia, CA, USA) with $0^{\circ}$ angulations, $7^{\circ}$ torque, and 0.022-inch archwire slots were used. A digital caliper was used to determine the bracket base surface areas as $11.9 \mathrm{~mm}^{2}$ and $10.6 \mathrm{~mm}^{2}$ for the COBs and MOBs (Clarity, Victory series, 3M Unitek, Monrovia, CA, USA), respectively.

After surface treatment of the bracket base, the adhesive resin paste (Transbond XT, 3M ESPE Dental Products, CA, USA) was applied and the bracket seated on the surface of the buccal surface of the provisional crown by a clinician. The excess adhesive resin was eliminated with a tip of explorer prior polymerization with a light cure (Ultralux, DabiAtlante, São Paulo, Brazil) from proximal directions for 20 seconds; each direction for each bracket. Then, a distilled water bath was used to store the samples at $37^{\circ} \mathrm{C}$. After 24 hours, the samples were exposed to thermocycling process for three cycles between $5^{\circ} \mathrm{C}$ and $55^{\circ} \mathrm{C}$ with a 20 -second dwell time and a 5-second transit time.

\section{Shear Bond Strength Test}

A universal testing device with a $5 \mathrm{kN}$ load cell (Lloyd Instruments PIc, Fareham, Hampshire, UK) was used to perform the SBS test (Fig. 1). A crosshead speed of $1 \mathrm{~mm} /$ minute was used to stress the samples in an occluso-gingival direction. ${ }^{10}$ The maximum load required to debond was reported in Newtons and later converted to megapascals (MPa). To evaluate the bond failure interface, the debonded bracket bases were inspected under an optical microscope at $16 \times$ magnification (Fig. 2). After debonding, the ARI was used to record the residual adhesive on the bracket base as follows: $0=$ no adhesive left on the bracket, $1=$ less than $50 \%$ of adhesive left on the bracket, $2=$ more than $50 \%$ of adhesive left on the bracket, and $3=100 \%$ of adhesive left on the bracket. ${ }^{20}$ When adhesive failure occurred at the cement-provisional material interface, it is scored as 0 or 1 according to this index. However, cohesive failure of the cement or bracket base-cement interface was scored as 2 or 3.

\section{Statistical Analysis}

The SPSS 11.0 software for Windows (SPSS Inc., Chicago, IL, USA) was used to perform the statistics. The two-way ANOVA was used to analyze the SBS data ( $\mathrm{MPa}$ ) while multiple comparisons were done via Tukey HSD post hoc tests. $p$ values $<0.05$ were considered statistically significant.

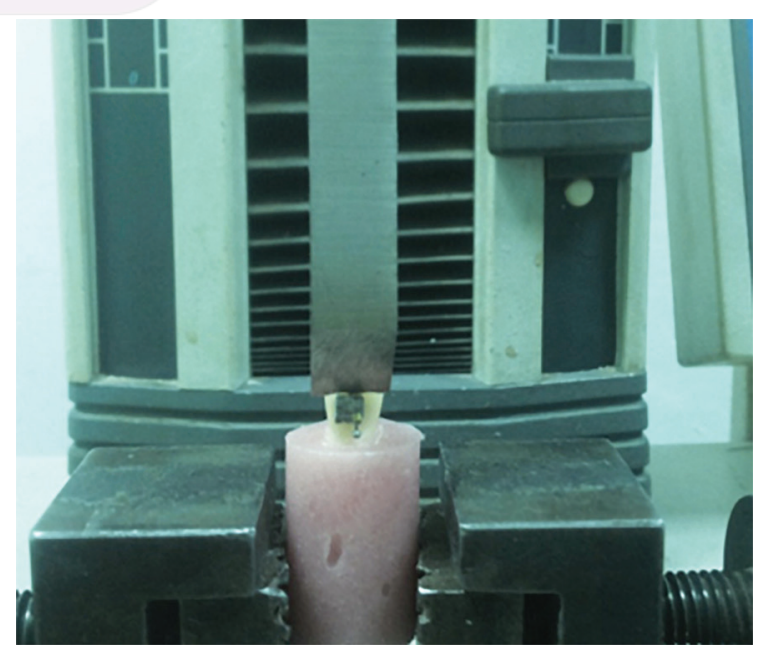

Fig. 1: Experimental set-up for the shear bond strength test with the tooth in position and the load applied through the universal testing machine 


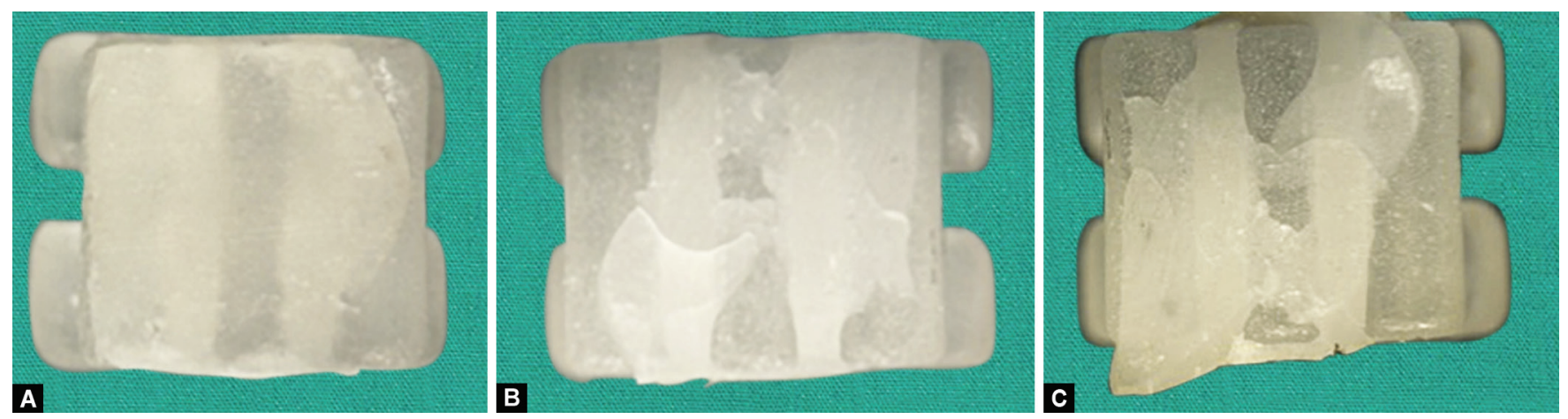

Figs 2A to C: Residual adhesive on the bracket. Adhesive remnant index scores represented as: (A) Score 1; (B) Score 2; (C) Score 3

Table 1: Shear bond strength mean values $(\mathrm{MPa}) \pm$ standard deviations of different experimental groups. Identical superscript letters in the same column indicate no significant difference (Tukey's test, $\alpha=0.05$ )

\begin{tabular}{llcc}
\hline Groups & Thermocycling & Ceramic bracket & Metal bracket \\
\hline Group CO & No & $9.6 \pm 1.4^{\mathrm{a}}$ & $7.7 \pm 2.3^{\mathrm{A}}$ \\
& Yes & $6.2 \pm 1.1^{\mathrm{b}}$ & $3.9 \pm 1.4^{\mathrm{B}}$ \\
Group HF & No & $17.8 \pm 2.1^{\mathrm{C}}$ & $15.5 \pm 1.6^{\mathrm{C}}$ \\
& Yes & $13.8 \pm 1.3^{\mathrm{d}}$ & $12.8 \pm 1.2^{\mathrm{D}}$ \\
Group SA & No & $17.2 \pm 1.4^{\mathrm{c}}$ & $15 \pm 1.2^{\mathrm{C}}$ \\
& Yes & $12.1 \pm 1.8^{\mathrm{e}}$ & $11.2 \pm 1.6^{\mathrm{E}}$ \\
\hline
\end{tabular}

Table 2: Percentage scores for the adhesive remnant index of all groups $(n=10)$

\begin{tabular}{|c|c|c|c|c|c|c|c|c|c|}
\hline \multirow[b]{2}{*}{ Groups } & \multirow[b]{2}{*}{ Thermocycling } & \multicolumn{4}{|c|}{ Ceramic brackets } & \multicolumn{4}{|c|}{ Metal brackets } \\
\hline & & Score 0 & Score 1 & Score 2 & Score 3 & Score 0 & Score 1 & Score 2 & Score 3 \\
\hline \multirow[t]{2}{*}{ Group CO } & No & 0 & 10 & 20 & 70 & 10 & 30 & 10 & 50 \\
\hline & Yes & 0 & 20 & 10 & 70 & 10 & 30 & 50 & 10 \\
\hline \multirow[t]{2}{*}{ Group HF } & No & 0 & 40 & 40 & 20 & 10 & 50 & 40 & 0 \\
\hline & Yes & 0 & 40 & 50 & 10 & 0 & 50 & 50 & 0 \\
\hline \multirow[t]{2}{*}{ Group SA } & No & 0 & 40 & 40 & 20 & 10 & 40 & 50 & 0 \\
\hline & Yes & 0 & 50 & 40 & 10 & 0 & 50 & 40 & 10 \\
\hline
\end{tabular}

\section{Results}

Descriptive statistics for all subgroups are shown in Table 1. Regarding the effect of thermocycling on SBS of the tested specimens, the two-way ANOVA showed significant differences among the subgroups of each bracket types. Within the COBs subgroups, the highest SBS value $(17.8 \pm 2.1,13.8 \pm 1.3 \mathrm{MPa})$ was obtained from the HF-treated group, and the lowest value $(9.6 \pm 1.4,6.2 \pm 1.1 \mathrm{MPa})$ was obtained from the CO group while the SA-treated group showed no significant difference $(p<0.05)$ from the HF-treated group $(17.2 \pm 1.4,13.3 \pm 1.2 \mathrm{MPa})$. For the MOBs, the values were $(7.7 \pm 2.3,3.9 \pm 1.4 \mathrm{MPa})$ for the CO group, $(15.5 \pm$ $1.6,12.8 \pm 1.2 \mathrm{MPa})$ for the HF group, and $(15 \pm 1.2,11.2 \pm 1.6 \mathrm{MPa})$ for the SA group.

Irrespective to the type of surface treatment used for indirect composite provisional restoration, bond strength values of metal brackets showed statically significant difference compared to ceramic bracket bonding $(p<0.05)$. The modes of failure for bracket types are shown in Table 2 . There were more failures at the bracket-adhesive interface in the MOBs group compared to the COBs group.

\section{Discussion}

The success of orthodontic treatment with fixed appliances depends, within other factors, on an accurate bracket positioning and long-term retention of these parts. The time spent during the bracket bonding is an important factor in the treatment cost and the necessity of rebonding brackets can retard the progress of treatment. The current research aimed to improve the bonding procedure to long-term provisional restorations by minimizing the time needed during bonding and debonding without endangering the ability to maintain a clinically useful bond strength.

Indirect composite material is recommended as a provisional crown in cases requires long-term prosthetic and orthodontic treatments, based on its superior mechanical strength compared with other provisional restorative materials. ${ }^{2}$ Their strength and stability are critical as fractures may occur during the treatment. Ideally, the bond strength between the brackets and the provisional material should be strong to avoid bracket debonding during the treatment; however, the bracket should be easily removed by the end of the treatment.

During the treatment, it is hard to define the magnitude of the bond strength required to withstand active orthodontic forces 
without bracket failure in different oral situations. ${ }^{21}$ However, it was reported that the estimated SBS range of 6.5-10 MPa is essential to maintain the bond of OBs to teeth. ${ }^{20,22-25}$ In this study, the mean SBS values of the surface-treated groups were within the clinically acceptable range of bond strengths.

Thermocycling intends to thermally stress the adhesive joint interface. Al Jabbari et al. ${ }^{15}$ reported that microleakage can occur due to fatigue of the joints between the adhesive and resin restoration since both have a different thermal coefficient of expansion, which leads to different volumetric changes during temperature fluctuations. The results indicated that $10 \% \mathrm{HF}$ etching for 1 minute was effective for the bonding of OBs to indirect composites before and after thermocycling as compared to SA-treated groups. This finding is consistent with a study conducted by Trajtenberg and Powers who showed that the use of $\mathrm{HF}$ etching was more effective for bonding to indirect composite restorations. ${ }^{12}$

When the indirect composite was exposed to hydrofluoric acid, the smear layer is eliminated and the surface becomes irregular and retentive for bonding. Another recommended method for improving bonding to composite provisional crowns is abrasion by airborne particles followed by silane coupling agent application. ${ }^{12-15}$ Many researchers concluded that airborne particle abrasion enhances roughness on the composite via non-selective degradation, thus producing microroughness, exterminates the resin matrix and maintaining filler particles on the surface for silane application. ${ }^{13,16}$

The mean value of the SBS test was constantly higher with the COBs than the MOBs. This may be due to that the relative translucency of COBs allows the transmission of curing light to the cement and subsequently, superior polymerization occurs. According to Al-Saleh and El-Mowafy, most failures arose at the resin-bracket interface when a light-cured cement was used. ${ }^{23}$ This could be as a result of incomplete polymerization of the resin cement below MOBs as the curing light was unable to penetrate through them. ${ }^{26}$

In this study, the failure pattern of debonded brackets was studied through visual inspection. Most of the failures in the MOBs group classified with ARI scores 1 and 2 demonstrating that all or more than half of the cement sustained on the provisional crown. However the failure pattern in the COBs groups, were ARI scores 1, 2 and 3. Adhesive failure was seen at the indirect composite-adhesive interface (ARI score 0 ) and at the bracket-adhesive interface (ARI score 3 ). Nearly cohesive failures were found within the indirect composite and also within the adhesive (ARI scores 1 and 2). Cohesive failure within the indirect composite could imply that the bond between the indirect composite and the adhesive resin was stronger than the composite itself. Thurmond et al. ${ }^{27}$ reported that cohesive fractures in the restorative material can occur when the values of the bond strength between the restorations and the adhesive resin exceeded $13 \mathrm{MPa}$. Since the difference between both HFand SA-treated groups was not significant and both results are within the clinically acceptable ranges, HF etching could be better than airborne particle abrasion. It is recommended to use the HF gel to overcome the harmful effects of HF solution and to prohibit its volatilization or dripping. Moreover, further investigations are required to assess the effect of different HF acid concentrations and etching time to achieve the appropriate procedures for clinical application.

\section{Conclusion}

The MOBs and COBs bonded to indirect composite provisional crowns can tolerate clinically acceptable orthodontic forces. Bonding of MOBs and COBs to the indirect composite can be increased when bonded surfaces were treated with either hydrofluoric acid or sandblasted followed by silane application. COBs displayed greater mean SBS when compared with MOBs.

\section{References}

1. Chay SH, Wong SL, Mohamed N, et al. Effects of surface treatment and aging on the bond strength of orthodontic brackets to provisional materials. Am J Orthod Dentofacial Orthop 2007;132(5):577.e7-611. e7. DOI: 10.1016/j.ajodo.2004.01.024.

2. Rambhia S, Heshmati R, Dhuru V, et al. Shear bond strength of orthodontic brackets bonded to provisional crown materials utilizing two different adhesives. Angle Orthod 2009;79(4):784-789. DOI: 10.2319/060908-298.1.

3. Vallittu PK. The effect of glass fiber reinforcement on the fracture resistance of a provisional fixed partial denture. J Prosthet Dent 1998;79(2):125-130. DOI: 10.1016/S0022-3913(98)70204-5.

4. Galindo D, Soltys JL, Graser GN. Long-term reinforced fixed provisional restorations. J Prosthet Dent 1998;79(6):698-701. DOI: 10.1016/S0022-3913(98)70078-2.

5. Balkenhol M, Mautner MC, Ferger P, et al. Mechanical properties of provisional crown and bridge materials: chemical-curing vs dual-curing systems. J Dent. 2008;36(1):15-20. DOI: 10.1016/ j.jdent.2007.10.001.

6. Blakey R, Mah J. Effects of surface conditioning on the shear bond strength of orthodontic brackets bonded to temporary polycarbonate crowns. Am J Orthod Dentofacial Orthop 2010;138(1):72-78. DOI: 10.1016/j.ajodo.2008.08.030.

7. Dias FM, Pinzan-Vercelino CR, Tavares RR, et al. Evaluation of an alternative technique to optimize direct bonding of orthodontic brackets to temporary crowns. Dental Press J Orthod 2015;20(4): 57-62. DOI: 10.1590/2176-9451.20.4.057-062.oar.

8. Haselton DR, Diaz-Arnold AM, Vargas MA. Flexural strength of provisional crown and fixed partial denture resins. J Prosthet Dent 2002;87(2):225-228. DOI: 10.1067/mpr.2002.121406.

9. Burke FJ, Sands P. Use of a novel resin composite crown as a longterm provisional. Dent Update 2009;36(8):481-484. DOI: 10.12968/ denu.2009.36.8.481, 486-7.

10. Hummel SK, Marker V, Pace L, et al. Surface treatment of indirect resin composite surfaces before cementation. J Prosthet Dent 1997;77(6):568-572. DOI: 10.1016/S0022-3913(97)70096-9.

11. D'Arcangelo C, Vanini L. Effect of three surface treatments on the adhesive properties of indirect composite restorations. J Adhes Dent 2007;9(3):319-326.

12. Trajtenberg CP, Powers JM. Effect of hydrofluoric acid on repair bond strength of a laboratory composite. Am J Dent 2004;17(3):173-176.

13. Fuentes MV, Ceballos L, González-López S. Bond strength of selfadhesive resin cements to different treated indirect composites. Clin Oral Investig 2013;17(3):717-724. DOI: 10.1007/s00784-012-0752-y.

14. Passos SP, Ozcan M, Vanderlei AD, et al. Bond strength durability of direct and indirect composite systems following surface conditioning for repair. J Adhes Dent 2007;9(5):443-447.

15. Al Jabbari YS, Al Taweel SM, Al Rifaiy M, et al. Effects of surface treatment and artificial aging on the shear bond strength of orthodontic brackets bonded to four different provisional restorations. Angle Orthod 2014;84(4):649-655. DOI: 10.2319/090313649.1.

16. Hori S, Minami H, Minesaki Y, et al. Effect of hydrofluoric acid etching on shear bond strength of an indirect resin composite to an adhesive cement. Dent Mater J 2008;27(4):515-522. DOI: 10.4012/dmj.27.515.

17. Maryanchik I, Brendlinger EJ, Fallis DW, et al. Shear bond strength of orthodontic brackets bonded to various esthetic pontic materials. 
Am J Orthod Dentofacial Orthop 2010;137(5):684-689. DOI: 10.1016/ j.ajodo.2008.06.031.

18. Vicente A, Bravo LA, Romero M, et al. A comparison of the shear bond strength of a resin cement and two orthodontic resin adhesive systems. Angle Orthod 2005;75:109-113. DOI: 10.1043/0003-3219(2005)075<0109:ACOTSB >2.0.CO;2.

19. Bishara SE, Ostby AW, Laffoon JF, et al. Shear bond strength comparison of two adhesive systems following thermocycling. A new self-etch primer and a resin-modified glass ionomer. Angle Orthod 2007;77(2):337-341. DOI: 10.2319/0003-3219(2007)077[0337:SBSCOT ]2.0.CO;2.

20. Ozcan M, Finnema K, Ybema A. Evaluation of failure characteristics and bond strength after ceramic and polycarbonate bracket debonding: effect of bracket base silanization. Eur J Orthod 2008;30(2):176-182. DOI: 10.1093/ejo/cjm100.

21. Turner PJ. Successful bonding in orthodontics: 1. Dent Update 1996;23(9):366-370.
22. Lopez JI. Retentive shear strengths of various bonding attachment bases. Am J Orthod 1980;77(6):669-678. DOI: 10.1016/00029416(80)90158-X.

23. Al-Saleh M, El-Mowafy O. Bond strength of orthodontic brackets with new self-adhesive resin cements. Am J Orthod Dentofacial Orthop 2010;137(4):528-533. DOI: 10.1016/j.ajodo.2008.04.027.

24. Reynolds IR. A review of direct orthodontic bonding. British Journal of Orthodontics 1975;2(3):171-178.DOI: 10.1080/0301228X.1975.11743666.

25. Kinami H, Sugimura M, Sakuda $M$, et al. New type metal bracket for suppression of resin remaining in debonding. Dent Mater J 1990;9(1):25-35. DOI: 10.4012/dmj.9.25.

26. Swartz ML, Phillips RW, Rhodes B. Visible light-activated resins-depth of cure. J Am Dent Assoc 1983;106(5):634-637. DOI: 10.14219/jada. archive.1983.0140.

27. Thurmond JW, Barkmeier WW, Wilwerding TM. Effect of porcelain surface treatments on bond strengths of composite resin bonded to porcelain. J Prosthet Dent 1994;72(4):355-359. DOI: 10.1016/00223913(94)90553-3. 